\title{
ARTICLE
}

\section{Measurement of Gamma-Ray Pulse Height Spectrum Under p-Li Neutron Beam Using NaI(Tl) Scintillator With Pulse-Shape-Discrimination Technique}

\author{
So KAMADA* and Masashi TAKADA \\ Research Center for Radiation Emergency Medicine, National Institute of Radiological Sciences \\ 4-9-1, Anagawa, Inage-ku, Chiba 263-8555 Japan
}

\begin{abstract}
We measured gamma-ray pulse-height spectrum of quasi-mono energetic neutron beam produced by the $\mathrm{p}$-Li reaction by using a $\mathrm{NaI}(\mathrm{Tl})$ scintillation detector, which has 7.62-cm diameter and 7.62-cm length, with pulse-shape-discrimination and time of flight (TOF) methods in National Institute of Radiological Sciences, Chiba, Japan. Prompt gamma-ray events in the TOF distribution are used to confirm our particle discrimination method. We can measure gamma ray events separately from neutrons in the neutron field.
\end{abstract}

\section{KEYWORDS: Gamma ray, Neutron, Time-of-flight method, Cyclotron accelerator, Pulse-shap e-discrimination mehtod, $\mathrm{NaI}(\mathrm{Tl})$ scintillator}

\section{Introduction}

$\mathrm{Li}(\mathrm{p}, \mathrm{xn})$ is very useful nuclear reaction to produce monoenergetic neutrons, because of high neutron production yield, having mono-energy neutron spectrum. This monoenergy neutron source is suitable to calibrate neutron dosimeters, for experiments of single event upsets of semi conductor devices, and several researches for neutron energies. In these experiments using p-Li neutron source, we have to subtract the effects by gamma rays. We are able to reduce the gamma ray background from interactions with neutrons and accelerator components, by appropriate shielding arrangement. However, it is impossible to neglect the gamma rays from the lithium target. In order to evaluate target gamma-ray background we have to separate gamma-rays events from neutron events. Gamma rays from target are emitted promptly, if the accelerator is driven on pulse mode, the difference of the flight speed of gamma rays and neutrons, we can measure by using the time of flight (TOF) method. However the p-Li reactions emit low energy gamma rays relative to neutrons, we cannot measure the prompt gamma rays with only TOF measurement. Hence, the particle discrimination is required in the gamma rays measurement under the $\mathrm{p}$-Li neutron field.

The differences of scintillator's pulse shape to several particles are known many years ago. It is very well known to use organic liquid scintillation detector to distinguish the gamma rays and neutrons by using pulse shape discrimination method. However organic liquid scintillator is composed with low atomic number materials. It is advantages in gamma-ray measurement because the cross sections of electron-gamma ray are low. To measure the gamma rays sufficiently in statistics, we utilized an $\mathrm{NaI}(\mathrm{Tl})$ scintillation detector, because of high detection efficiencies, good energy resolutions among the several scintillators.

*Corresponding author, Email: s_kamada@nirs.go.jp

(C) 2012Atomic Energy Society of Japan All Rights Reserved
Moreover the $\mathrm{NaI}(\mathrm{Tl})$ scintillator is the most available scintillators in the world. Eby and Jentschke ${ }^{1)}$ investigated the fluorescent response of $\mathrm{NaI}(\mathrm{Tl})$ to different types of particles, such as deuteron, alpha, and gamma rays and observed that the decay time to gamma rays is longer than that to proton, deuteron and alpha particle. Owen ${ }^{2)}$ applied the differences of pulse shape to the particle discrimination. Share ${ }^{3)}$ observed the distinguishing of particle discrimination by using an $\mathrm{NaI}(\mathrm{Tl})$ scintillation detector under the tens of $\mathrm{MeV}$ neutrons. We apply the pulse shape discrimination (PSD) to separation gamma-rays events from neutrons in the p-Li neutron field.

\section{Experiment \\ 1. Detector}

For gamma-ray measurement, We utilized a $\mathrm{NaI}(\mathrm{Tl})$ scintillation detector, whose size was $7.62-\mathrm{cm}$ in diameter and $7.62-\mathrm{cm}$ in length. The length of this scintillator enabled us to measure the gamma rays up to $150 \mathrm{MeV}$, because the range of electrons of $150 \mathrm{MeV}$ in $\mathrm{NaI}(\mathrm{Tl})$ crystals are around $7 \mathrm{~cm}^{4)}$. The detector was assembled with photomultiplier, R7195 manufactured by Hamamatsu Photonics Japan. The photomultiplier tube was surrounded by magnetic shield. The detector bias applied to photomultiplier was $-2000 \mathrm{~V}$. $\mathrm{NaI}(\mathrm{Tl})$ scintillation detector has relatively higher $\mathrm{Z}$ than that of organic liquid scintillator, so photo-electric peak can be formed. $\mathrm{NaI}(\mathrm{Tl})$ crystal has a good energy resolution $(\sim 7.5 \%$ for $662 \mathrm{keV}$ gamma ray) in scintillation crystals. Moreover, gamma rays measurement using $\mathrm{NaI}(\mathrm{Tl})$ scintillator is well known to obtain energy spectrum by using unfolding method easily due to its peak to total ratio.

\section{2.p-Li Neutron field and experimental procedure}

We carried out the experiment at the neutron fields produced by the ${ }^{7} \mathrm{Li}(\mathrm{p}, \mathrm{xn})$ reaction of $40 \mathrm{MeV}$ proton beams, at cyclotron facility of National Institute of Radiological Sciences (NIRS) cyclotron facility in Chiba, Japan ${ }^{5}$. The 
accelerator produce pulsed proton beam (1.5 ns width in FWHM, $14.85 \mathrm{MHz}$ repetition rate). ${ }^{7} \mathrm{Li}(\mathrm{p}, \mathrm{xn})$ reaction create high-energy sharp peak at tens of $\mathrm{MeV}$. This neutron energy spectrum comprises of high-energy peak and continuum $^{6}$. In the experiments, $40 \mathrm{MeV}$ proton beams loss $2 \mathrm{MeV}$ in the lithium target with $4.7 \mathrm{~mm}$ thickness. Proton beams through the lithium target were bent by the bending magnet and down to carbon beam dump. We obtained incident proton beam fluxes from integration of proton beams into the beam dump. Lithium and copper targets are used for neutron and prompt gamma production, respectively. The thickness of copper target was $10 \mathrm{~mm}$ to stop the proton beams in the target. Targets, which were installed in the target vacuum chamber on beam line to protect lithium metal from humidity, were controlled remotely from out of the beam irradiation room. The scintillation detector was set 5.0 $\mathrm{m}$ downstream from the lithium target. The signals from the detector were fed into the electric circuits and then processed with the KODAQ data acquisition system ${ }^{7)}$.

\section{Pulse Shape Discrimination method}

We utilized charge-integration type ADC, LeCroy 2249W (manufactured by LeCroy, Chestnut Ridge, NY, USA) for pulse-shape-discrimination method. This ADC integrates signal charge during total and tail gates, as shown in Figure 1. Solid line and dashed line in Fig. 1 indicate the signal due to recoil by neutron and gamma rays, respectively. The gates open $1 \mu$ sec widths acquiring a signal peak, and the gates are delayed from signal rise time at 270 nano-sec. ADC values integrating signals during the total- and tail- gates are called as total and tail components, respectively.

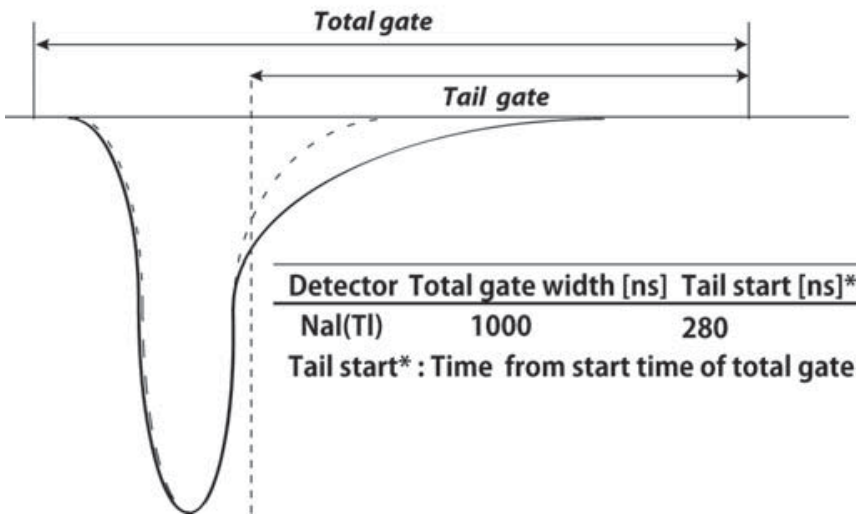

Fig. 1 Correlation between scintillation signals and gates of charge integration for total and tail components.

Figure 2 shows a two-dimensional pulse height distribution, measured using $\mathrm{NaI}(\mathrm{Tl})$ scintillators in the $\mathrm{p}-\mathrm{Li}$ neutron beam. The horizontal and vertical axes indicate pulse heights of total and tail components, respectively in relative values. The neutron events discriminated in Fig.2 was used to obtain measured using $\mathrm{NaI}(\mathrm{Tl})$ scintillators in the $\mathrm{p}-\mathrm{Li}$ neutron beam. The horizontal and vertical axes indicate pulse heights of total and tail components, respectively in relative values. The electron line due to gamma-ray events and proton-, deuteron- and alpha-particle- lines due to neutron events discriminated in Fig. 2 was used to obtain the gamma-ray events. We measured gamma-ray sources of ${ }^{22} \mathrm{Na}$, ${ }^{60} \mathrm{Co}$, and ${ }^{137} \mathrm{Cs}$ and $\mathrm{Am}-\mathrm{Be}$ neutron source to confirm our discrimination technique using the $\mathrm{NaI}(\mathrm{Tl})$ scintillator. Figure 3 compared two-dimensional plots of the p-Li neutron beam and ${ }^{60} \mathrm{Co}$ gamma-ray source. We can observe the gray plots by ${ }^{60} \mathrm{Co}$ distributing over the gamma-rays domain. From this comparison, we confirmed gamma ray events in the pulse-shape-discrimination plot, measured of neutrons and gamma rays, simultaneously. Saturated events, indicated by an arrow in the Fig. 2 and 3 may be created by cosmic muons.

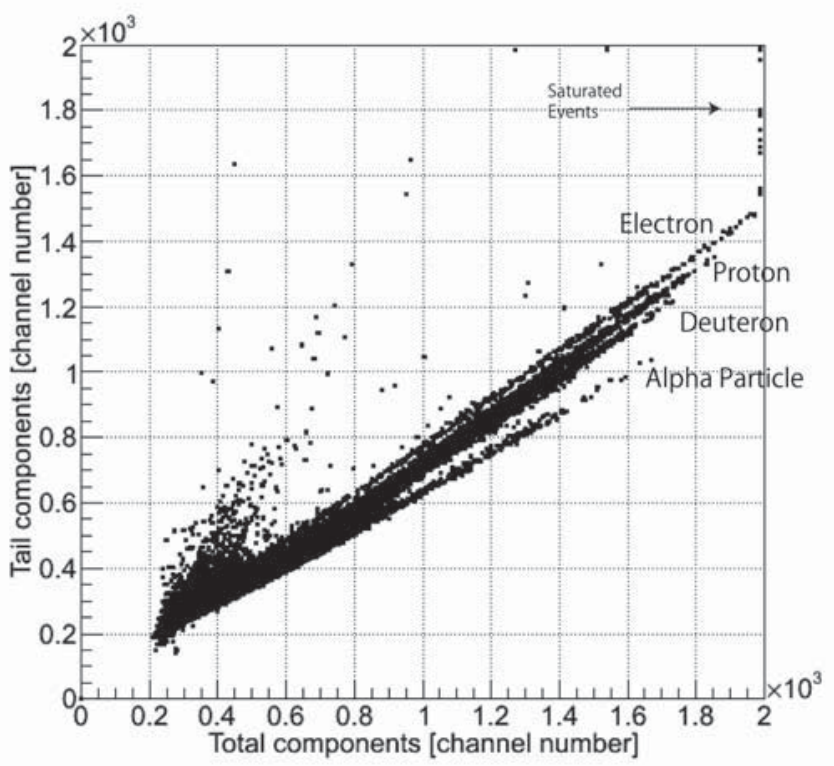

Fig. 2 Two-dimensional plots of total and tail components using $\mathrm{NaI}$ (Tl) scintillation detectors, respectively.

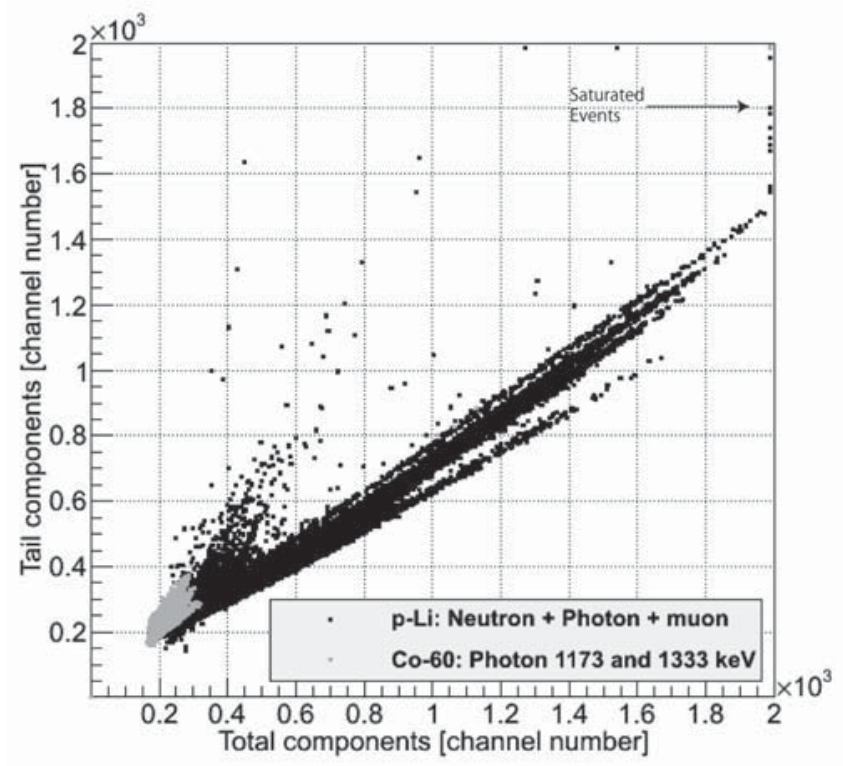

Fig. 3 Two-dimensional plot of particle discrimination of total and tail components, measured using the $\mathrm{NaI}(\mathrm{Tl})$ scintillator. Black and grey dots show events measured in the $\mathrm{p}$ - $\mathrm{Li}$ neutron beams and ${ }^{60} \mathrm{Co}$ gamma-ray source, respectively.

\section{Event selection using Time of flight measurement}

We also acquired TOF signals to confirm gamma-ray and 
neutron events using the pulse shape discrimination. And then, we determined the position of the prompt gamma-ray peak by using a copper target, because p-Li emits low-energy gamma rays, so gamma-rays events are covered with low energy neutron events. Figure 4(a) shows a TOF distribution measured using the $\mathrm{NaI}(\mathrm{Tl})$ scintillator in the p-Li neutron beam. The quasi-monoenergetic neutron peak is observed at 1700 (ch.) of TOF distribution. We measured prompt gamma ray to obtain time origin of nuclear reaction by bombarding $40 \mathrm{MeV}$ proton beams into the thick copper target. The prompt gamma rays are observed at 1000 (ch.) in the Fig.4-(b) The prompt gamma-ray events are selected from the region of interest around the gamma-flash, as described with lines.

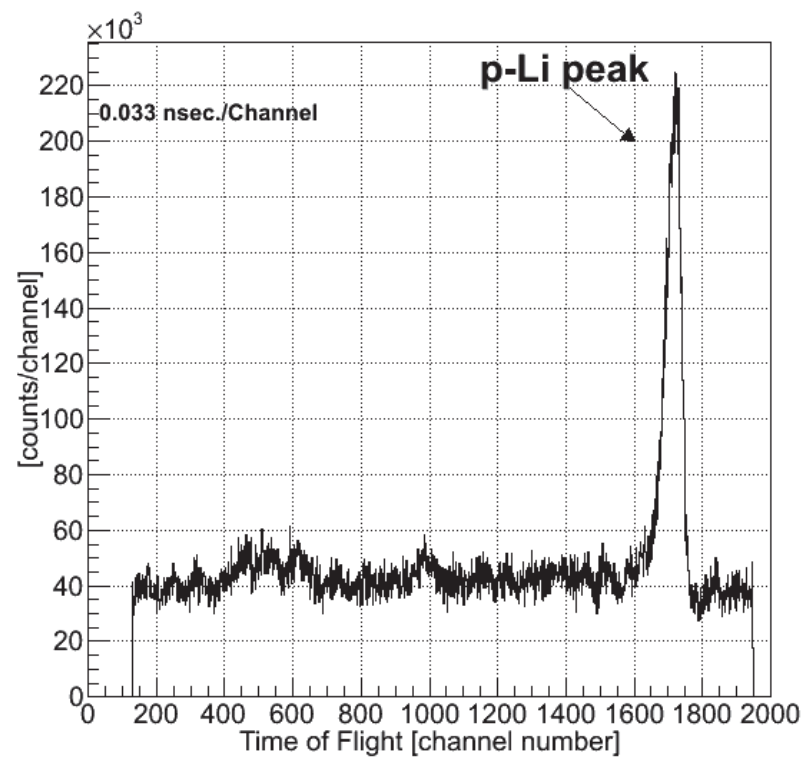

Fig. 4(a) TOF distribution measured by using the $\mathrm{NaI}(\mathrm{Tl})$ scintillator in the p-Li neutron beam and $\mathrm{p}-\mathrm{Cu}$ beam in Fig.4-(a) and in Fig.4-(b), respectively.

\section{Results}

We applied the gamma-ray gate by surrounding the gamma-ray line in the fig.2. Then, we show the TOF distributions separated by gamma-rays gate and neutron-gate in Figure 5. We can observe the gamma-ray peak in the TOF distribution by using the PSD method. We can observe the prompt gamma-ray peak whose position agrees with the position of prompt gamma-ray peak in the TOF distribution measured in the $\mathrm{p}-\mathrm{Cu}$ beam. Then we selected prompt gamma-ray region in the TOF distribution, and obtained gamma-ray pulse height spectrum by selecting gamma-ray events from neutrons using the pulse-shape-discrimination plot and gamma-ray peak in the TOF distributions measured in the p-Li neutron beam, as shown in Fig. 6. Horizontal and vertical axes indicate light output of the $\mathrm{NaI}(\mathrm{Tl})$ scintillator as $\mathrm{MeVee}$ (equivalent to $1 \mathrm{MeV}$ electron light output) unit and the number of count per channel, respectively. In this paper, we assumed the statistical uncertainties only, and the uncertainties are indicated as error bars in the Fig. 7. We discriminate gamma ray events from 2.5 from $12 \mathrm{MeVee}$ light output. This spectrum has characteristic structures, which is the edge around $6.5 \mathrm{MeV}$ and the peak around 7.5
$\mathrm{MeV}$. These are estimated to be gamma-rays transitions from the third $\left(\mathrm{Ex}=6.68 \mathrm{MeV}, \mathrm{J}^{\pi}=5 / 2-\right)$ state and the forth (Ex $=0.746 \mathrm{MeV}, \mathrm{J} \pi=5 / 2-)$ to the ground $\left(\mathrm{Ex}=0 \mathrm{MeV}, \mathrm{J}^{\pi}=\right.$ $3 / 2^{-}$) state of ${ }^{7} \mathrm{Li}$ residual from inelastic scattering of ${ }^{7} \mathrm{Li}$ with incident protons $\left({ }^{7} \mathrm{Li}\left(\mathrm{p}, \mathrm{p}^{\prime}\right)^{7} \mathrm{Li} \text { reactions }\right)^{8}$.

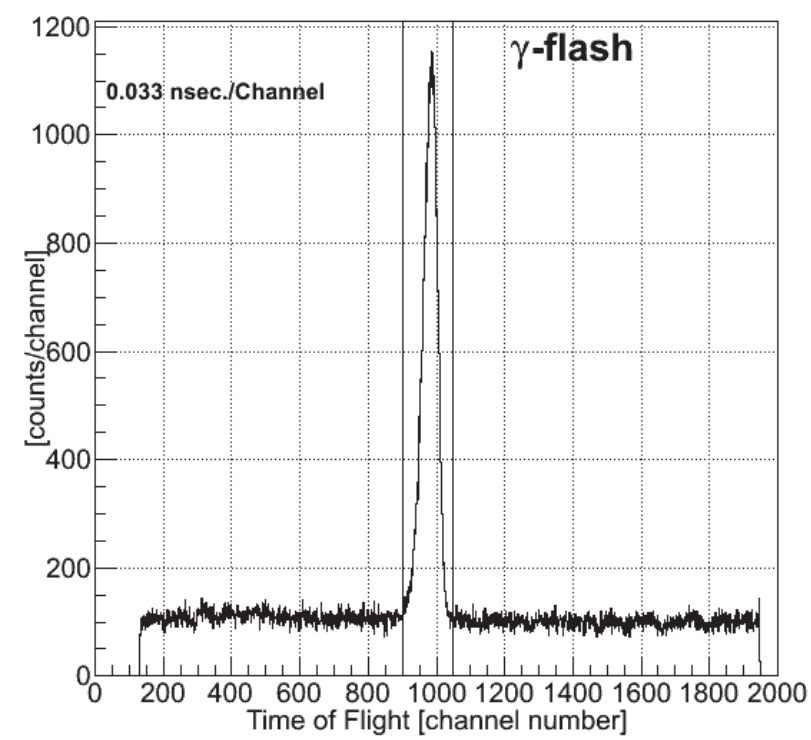

Fig. 4(b) TOF distribution measured by using the $\mathrm{NaI}(\mathrm{Tl})$ scintillator in the $\mathrm{p}-\mathrm{Cu}$ beam

Tac

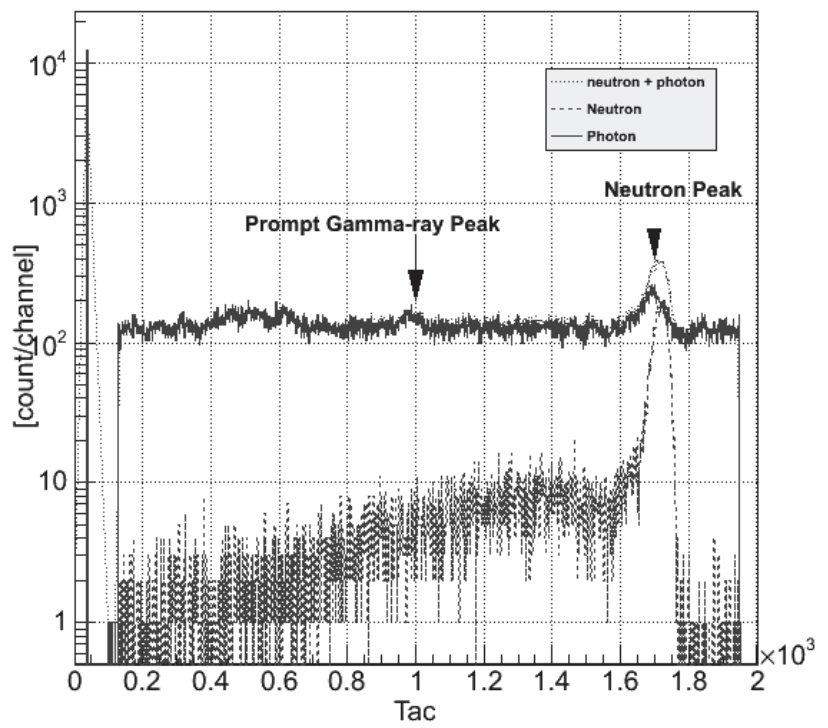

Fig. 5 TOF distribution measured by using the $\mathrm{NaI}(\mathrm{Tl})$ scintillator in the p-Li neutron beam. Arrows in this figure indicate the positions of the prompt gamma-ray peak and neutron peak, respectively. And time-per-channel in this figure is $0.033 \mathrm{nsec} /$ Channel. The dots line, dash line, and solid line indicate TOF distributions by without gate, and with neutron gate, and gammaray gate, respectively.

\section{Summary}

We have obtained two-dimensional plot of pulse-shape discrimination using the $\mathrm{NaI}(\mathrm{Tl})$ scintillation detector. From the measurement of the gamma ray sources and the $\mathrm{p}-\mathrm{Li}$ 
With Pulse-Shape-Discrimination Technique

neutron beams, we confirmed that pulse-shape-discrimination method using the $\mathrm{NaI}(\mathrm{Tl})$ scintillator had an ability of separation between gamma rays and neutrons. We measured the gamma-ray pulse height spectrum in the quasi-monoenergetic neutron source produced by $40 \mathrm{MeV}$ proton beam by selecting the gamma ray events using the pulse-shape-discrimination method. We will obtain gamma-ray energy spectrum by unfolding method with the response functions for high-energy photons.

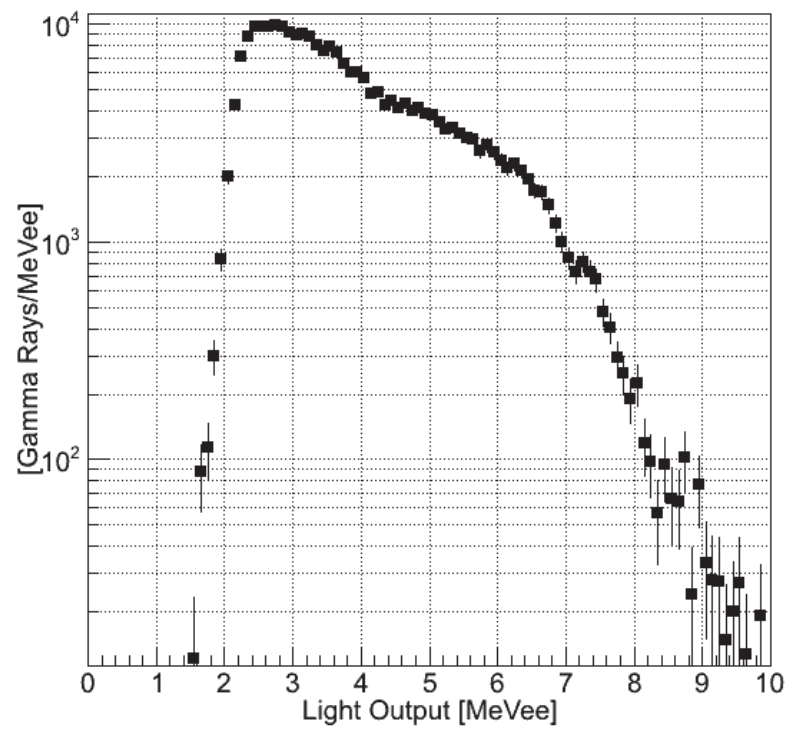

Fig. 6 Prompt gamma-ray pulse height spectrum, separated with pulse shape discrimination method and TOF method measured using the $\mathrm{NaI}(\mathrm{Tl})$ scintillation detector in the $\mathrm{p}-\mathrm{Li}$ neutron beam.

\section{Acknowleagement}

We thank all cyclotron staff of National Institute of Radiological Sciences.

\section{References}

1) F. S. Eby and W. K. Jentschke, "Fluorescent Response of $\mathrm{NaI}(\mathrm{Tl})$ to Nuclear Radiations," Phys. Rev. 96, 911-920, (1954).

2) R. B. Owen, "Pulse Shape Discrimination - A survey of current Technoques," IRE Trans. Nucl. Sci. NS-9, p.285-203, (1962).

3) G. M. Share, J. D. Kurfess, and R. B. Theus, "Pulse-shape discrimination of High-energy neutrons and Gamma rays in NaI(Tl)," Nucl. Instrum. Methods, Vol.148, p.531-533, (1978).

4) M. J. Berger, J. S. Coursey, M. A. Zucker and J. Chang, "Stopping-Power and Range Tables for Electrons, Photons, and Helium Ions, NIST Standard Reference Database 124, (http://www.nist.gov/pml/data/star/index.cfm).

5) H. Ogawa, T. Yamada, Y. Kumamoto, Y. Sato, T. Hiramoto, "Status Report on the Nirs-Chiba Isochronous Cyclotron Facility," IEEE trans. Nucl. Sci. NS-26 (2). (1988).

6) C. J. Batty, B. E. Bonner, A. I. Kilvington, C. Tscharlär, L. E. Williams, A. S. Clough, "Intermediate energy neutron sources," Nucl. Instrum. Methods, Vol.68, 273-276, (1969).

7) K. Omata, Y. Fujita, N. Yoshikawa, M. Sekiguchi, and Y. Shida. A Data Acquisition system based on a personal computer, INS-Rep.-884, Institute for Nuclear Study, University of Tokyo, (1991).

8) R. B. Firestone, V. S. Shirley, C. M. Baglin, S. Y. F. Chu, J. Zipkin, Table of Isotopes, 8th ed., John Wiley \& Sons, New York (1998) 